\title{
Anti-metastatic Efficacy of Traditional Chinese Medicine (TCM) Ginsenoside Conjugated to a VEFGR-3 Antibody on Human Gastric Cancer in an Orthotopic Mouse Model
}

\author{
XIAOJUN DAI ${ }^{1,2,3^{*}}$, DONGMEI LIU ${ }^{1 *}$, MOXIANG LIU ${ }^{1}$, XIAOCHUN ZHANG ${ }^{3}$, WEIMING WANG ${ }^{4}$, \\ FENG JIN $^{1}$, YAYUN QIAN ${ }^{1}$, XUANYI WANG ${ }^{1}$, JINGJING ZHAO ${ }^{2}$, YONGJIAN WU ${ }^{3}$, FANGFANG XIONG ${ }^{2}$, \\ NA CHANG ${ }^{2}$, YU SUN ${ }^{6}$, ZHIJIAN YANG ${ }^{5,6}$, ROBERT M. HOFFMAN ${ }^{5,7}$ and YANQING LIU ${ }^{1}$ \\ ${ }^{1}$ Clinical Traditional Chinese Medical College, Yangzhou University, Yangzhou, P.R. China; \\ ${ }^{2}$ Affiliated Yangzhou Hospital of Nanjing University of Traditional Chinese Medicine, Yangzhou, P.R. China; \\ ${ }^{3}$ Yangzhou Traditional Chinese Medicine Hospital, Yangzhou, P.R. China; \\ ${ }^{4}$ Yixing People's Hospital, Yixing, P.R. China; \\ ${ }^{5}$ AntiCancer, Inc., San Diego, CA, U.S.A.; \\ ${ }^{6}$ Origin Biosciences Inc., Nanjing, P.R. China; \\ ${ }^{7}$ Department of Surgery, University of California, San Diego, CA, U.S.A.
}

\begin{abstract}
Background/Aim: The aim of the present study was to investigate the efficacy of a traditional Chinese medicine (TCM), VEGFR-3 antibody-conjugated ginsenoside $R_{3}$ nanoemulsion (VRIN), targeting lymphangiogenesis, on the inhibition of tumor growth and metastasis in an orthotopic mouse model of human gastric cancer. Materials and Methods: An orthotopic nude-mouse model of gastric cancer was established with the red fluorescent protein (RFP)-expressing human gastric cancer cell line NUGC-4-RFP. The tumorbearing mice were treated with vehicle $(0.2 \mathrm{ml}$ normal saline every other day, iv), 5-FU (20 mg/kg once a week, i.p.) and VRIN (1 mg/kg every other day, i.v.). Real-time fluorescence imaging was performed to assess tumor inhibition in each group. Metastasis was evaluated by open fluorescence imaging at autopsy. The expression of lymphangiogenesis-related factors $V E G F-C, V E D F-D$ and VEGFR-3 in the tumors were analyzed
\end{abstract}

This article is freely accessible online.

*These Authors contributed equally to this study.

Correspondence to: Yanqing Liu, Clinical Traditional Chinese Medical College, Yangzhou University, Yangzhou, Jiangsu, P.R. China. Tel: +86 51487937083, Fax: +86 51487937133, e-mail: liuyq@yzu.edu.cn or Robert M. Hoffman, Ph.D., AntiCancer Inc., 7917 Ostrow Street, San Diego, CA 92111, U.S.A. Tel: +1 8586542555, Fax: +1 8582684175, e-mail: all@anticancer.com

Key Words: Ginsenoside $\mathrm{Rg}_{3}$, immune-nanoemulsion, VEGFR-3, gastric cancer, orthotopic, nude mice, metastasis, red fluorescent protein, RFP, imaging, efficacy. by immunohistochemistry and real-time RCP. Results: VRIN and 5-FU significantly inhibited primary tumor growth as compared to vehicle control $(p<0.05)$. However, significant inhibition of lymph-node metastasis was only found in the VRIN-treated group $(p<0.05)$. The expression of VEGF-C, VEGF-D and VEGFR-3 in the tumor was suppressed by VRIN treatment $(p<0.05)$. Expression of VEGF-D and VEGFR-3 in the 5-FUtreated group was not significantly increased ( $p>0.05)$. No obvious toxicity was found in VRIN- and 5-FU-treated groups. Conclusion: Lymphangiogenesis-targeted ginsenoside $\mathrm{Rg}_{3}$ immune-nanoemulsion inhibited tumor growth and reduced lymphatic metastasis by suppressing expression of VEGF-C, VEGF-D and VEGFR-3 in an orthotopic mouse model of human gastric cancer. Our study demonstrates the potential of TCM as an effective targeted treatment for metastatic gastric cancer.

Gastric cancer (GC) is the most frequently occurring cancer in Southeast Asian countries $(1,2)$. Postoperative recurrence of GC is high in patients with advanced or metastatic disease $(3,4)$.

Lymph-node metastasis is an important prognostic factors for patients with GC (5-7). Vascular endothelial growth factor receptor (VEGFR)-3 is involved in lymphogeneous metastasis along with vascular endothelial growth factor $\mathrm{C}$ (VEGF-C) and D (VEGF-D) (8-10). VEGF-C and -D promote lymphangiogenesis and lymph-node metastasis in GC $(11,12)$.

Ginsenoside $\mathrm{Rg}_{3}$ is derived from the Chinese herb ginseng with a molecular formula of $\mathrm{C}_{42} \mathrm{H}_{72} \mathrm{O}_{13}$ and has been demonstrated to have anti-cancer activity (13-16). Ginsenoside $\mathrm{Rg}_{3}$ can inhibit cancer-cell proliferation and induces apoptosis (17). However, due to its poor aqueous solubility and low oral bioavailability, in vivo use of ginsenoside $\operatorname{Rg}_{3}$ has been limited. 
Nanoemulsions have shown benefits for drug delivery and availability (18-22). In the present study, we investigated the efficacy of a lymphangiogenesis-targeted ginsenoside $\mathrm{Rg}_{3}$ nanoemulsion (VRIN) in an orthotopic nude-mouse model of human gastric cancer.

\section{Materials and Methods}

Cell culture. The NUGC-4 human gastric cancer cell line expressing red fluorescent protein (RFP) was obtained from AntiCancer, Inc. (San Diego, CA, USA). The cell line was cultured in DMEM supplemented with 10\% fetal bovine serum (Hyclone, Logan, UT, USA) and penicilin/streptomycin (Gibco Life Technologies, Grand Island, $\mathrm{NY}, \mathrm{USA}$ ) at $37^{\circ} \mathrm{C}$ and $5 \% \mathrm{CO}_{2}$ atmosphere.

Animal care. BALB/C female nude mice (21), aged 4-6 weeks and weighing 20-25g, were purchased from the Yang Zhou University Laboratory Animal Center (SCXK, Su. 20120004). All mice were maintained in a HEPA-filtered environment at $24-25^{\circ} \mathrm{C}$ and humidity was maintained at $50-60 \%$. All animals were fed with autoclaved laboratory rodent diet. Animal experiments were approved by the Animal Committee of Nanjing Origin Biosciences, China (OB1211).

Orthotopic mouse model of gastric cancer. The NUGC-4-RFP human gastric-cancer cells were harvested at $80 \%$ confluence by digestion with $0.25 \%$ trypsin solution. Stock tumor was established by subcutaneously injecting NUGC-4-RFP cells $\left(5 \times 10^{6}\right)$ into the flank of nude in mice.

Tumors grown in nude mice were harvested at the exponential growth phase and resected under aseptic conditions. Strong RFP expression of the NUGC-4-RFP tumor tissue was confirmed by fluorescence microscopy. Necrotic tissues were removed and viable tissues were cut with scissors and minced into $1 \mathrm{~mm}^{3}$ pieces. The surgical orthotopic implantation (SOI) method followed published procedures (23). Animals were anesthetized by injection of $0.02 \mathrm{ml}$ of solution of $50 \%$ ketamine, $38 \%$ xylazine, and $12 \%$ acepromazine maleate. All surgical procedures and animal manipulations were conducted under aseptic conditions in a HEPA-filtered laminar-flow hood under a surgical microscope (Model YZ20P5, Shanghai Precision Instruments, Shanghai, China).

Treatment. VEGFR-3 antibody conjugated ginsenoside $\mathrm{Rg}_{3}$ immunenanoemulsion (VRIN) was synthesized by the Laboratory of Natural Product Drugs, Yang Zhou University, China using a polyethylene glycol (PEG) method according to a published protocol (18). Nanoemulsions were prepared using a two-step emulsification process: a first step to form the primary emulsions and a second step to form multiple nanoemulsions. The viscosity of VRIN was $1.09 \pm 0.03 \mathrm{mPa} \cdot \mathrm{s}$ The mean Zeta electric potential was $-11.8 \mathrm{mV}$. The mean particle diameter was $137.9 \mathrm{~nm}$.

Treatments were initiated 9 days after SOI. The mice were randomly divided into three groups of eight. Group 1 (vehicle control) received intravenous saline injection every other day at $0.2 \mathrm{ml} / \mathrm{mouse}$ for three weeks. Group 2 received intraperitoneal 5-FU once a week at $20 \mathrm{mg} / \mathrm{kg} / \mathrm{dose}$ for three weeks. Group 3 received intravenous VRIN injection every other day at $1 \mathrm{mg} / \mathrm{kg}$ for three weeks. Tumor growth was measured by whole-body fluorescence imaging (24) with a fluorescence stereo microscope (MZ650; Nanjing Optical Instruments Inc., Nanjing, China) equipped with bandpass HQ600/50 emission and HQ540/40 excitation filters (Chroma Technology, Brattleboro, VT, USA). Animal body weights and clinical signs were recorded over the course of the experiments. All animals were sacrificed 31 days after tumor implantation. At autopsy open fluorescence imaging was performed to examine metastases and then the tumor was removed and weighed. Images were processed and analyzed with the use of IMAGE PRO Plus 6.0 software (Media Cybernetics, Silver Spring, MD, USA).

Quantitative real-time polymerase chain reaction (RT-PCR). TRIzol Reagent (Invitrogen, USA) was used to extract the total RNA after tissues were homogenized using a homogenizer (PRO, PRO Scientific Inc., USA). Total RNA ( $1 \mu \mathrm{g}$ ) was reverse-transcribed to cDNA with olig $(\mathrm{dT})$ primer using the reverse transcription system (Promega, Madison, WI, USA). The following primers were used to amplify most of the coding region of VEGF-C (sense, 5'-TCCTCGGATGCTGGAGA TGAC-3', and antisense, 5'-TTGGCTGGGAAGAGTTTGTT-3'); VEGF-D (sense, 5'-GGACTCTCGCTCAGCATCCCATC-3', and antisense, 5'-CCACCTCCACGCACGTTTCTCTA-3'); VEGFR-3 (sense, 5'-CAGGAGGTGGTGTGGGATGAC-3', and antisense, 5'CAGGAAGGGGTTGGAAAGGAAGT-3') and GAPDH (sense, 5'GCACCGTCAAGGCTGAGAAC-3', and antisense, 5'-TGGTGAAGA CGCCAGTGGA-3')

Quantitative RT-PCR was performed on an ABI PRISM 7000 sequence-detection system (Applied Biosystems, USA). RT-PCR was carried out in a total volume of $30 \mu \mathrm{l}$, and the reaction-induced $1 \mathrm{X}$ buffer, $200 \mu \mathrm{M}$ dNTPs (Invitrogen, Carlsbad, CA, USA), 0.3 $\mu \mathrm{M}$ sense and anti-sense primer, 1U Takara ExTaq Hotstart taq (TaKaRa Biotechnology, Co., Ltd, Dalian, Liaoning Province, China) and $2 \mu \mathrm{l} \mathrm{cDNA}$. PCR started at $95^{\circ} \mathrm{C}$ for 2 minutes, followed by 40 cycles of amplification: denaturation at $94^{\circ} \mathrm{C}$ for 30 seconds, annealing at $58^{\circ} \mathrm{C}$ (for detection of GAPDH) or $55^{\circ} \mathrm{C}$ (for detection of VEGF-C) for 30 seconds and prolongation at $72^{\circ} \mathrm{C}$ for 1 minute. Gene expression was calculated using the $2^{-\Delta \Delta \mathrm{Ct}}$ method.

Immunohistochemistry. Formalin-fixed, paraffin-embedded tumor tissues were sectioned at $4 \mu \mathrm{m}$ thickness. Sections were deparaffinized using $100 \%$ xylene, and dehydrated with an ethanol gradient. Antigen retrieval was performed with autoclave treatment $\left(120^{\circ} \mathrm{C}\right.$ for $2 \mathrm{~min}$ in $1 \mathrm{mmol} / \mathrm{L}$ EDTA, pH 8.0). Incubation with a polyclonal antibody raised against VEGF-C, VEDF-D and VEGFR-3 (Abgent, San Diego, CA, USA) at a dilution 1:200, was performed overnight at $\mathrm{pH} 7.4$ and $4^{\circ} \mathrm{C}$. After washing with phosphate-buffered saline (PBS), sections were then incubated with secondary antibody (Dako REAL EnVision Detection System, Dako, Ely, Cambridgeshire, UK) for $30 \mathrm{~min}$ at room temperature. Color development was performed with 3, 3'diaminobenzidine (DAB). Nuclei were lightly counter-stained with hematoxylin. The slides were viewed at $\times 400$ magnification and positive cells were recognized by the appearance of brown stain. The expression level was quantified by the average optical density (AOD) of the positive cells in five fields per sample with ImagePro Plus 6.0 software (Media Cybernetics).

Statistics. Data are expressed as mean \pm standard deviation. All statistical analyses were performed using SPSS16.0 software (SPSS inc., Chicago, Illinois, USA). Data comparisons between two groups were performed using the Student's $t$-test. Fisher's exact test was used to compare the difference in the incidence of metastasis. A value of $p \leq 0.05$ was regarded as statistically significant. 

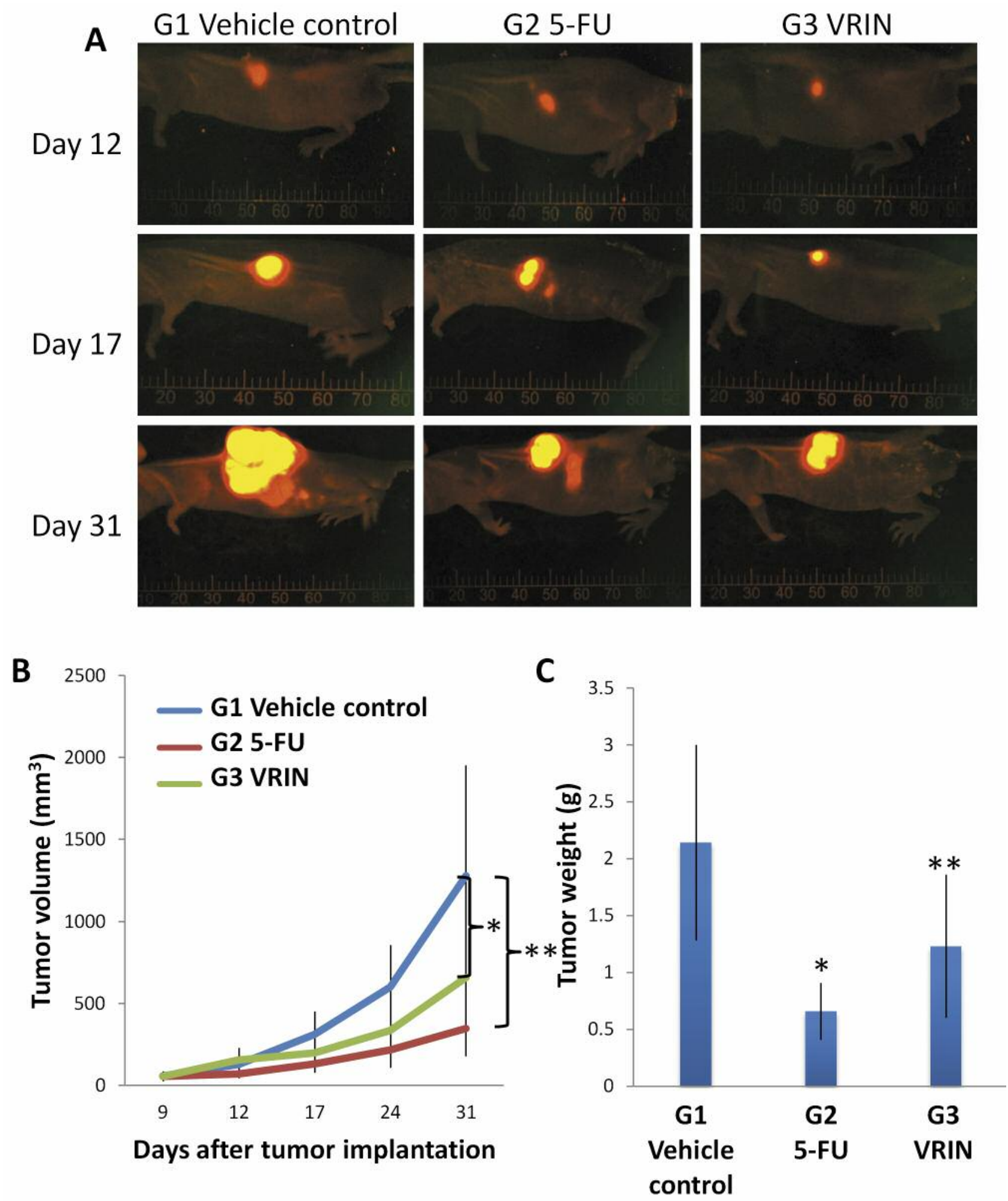

Figure 1. Efficacy of VRIN treatment on tumor growth in the NUGC-4-RFP gastric-cancer orthotopic mouse model. Tumor growth was monitored and quantified by real-time whole-body fluorescence imaging. (A) Sequential in vivo whole-body fluorescence imaging of tumors on day 12, 17 and 31 after tumor implantation in mice treated with vehicle, 5-FU or VRIN. (B) Tumor growth curves for mice treated with vehicle, 5-FU or VRIN. ${ }^{*} p=0.036 ; * *=0.031$. (C) Final tumor weights at the end of the study in treated mice. Tumors were resected and final tumor weight was determined at autopsy. $* p=0.004 ; * *=0.03$, compared to vehicle control. 

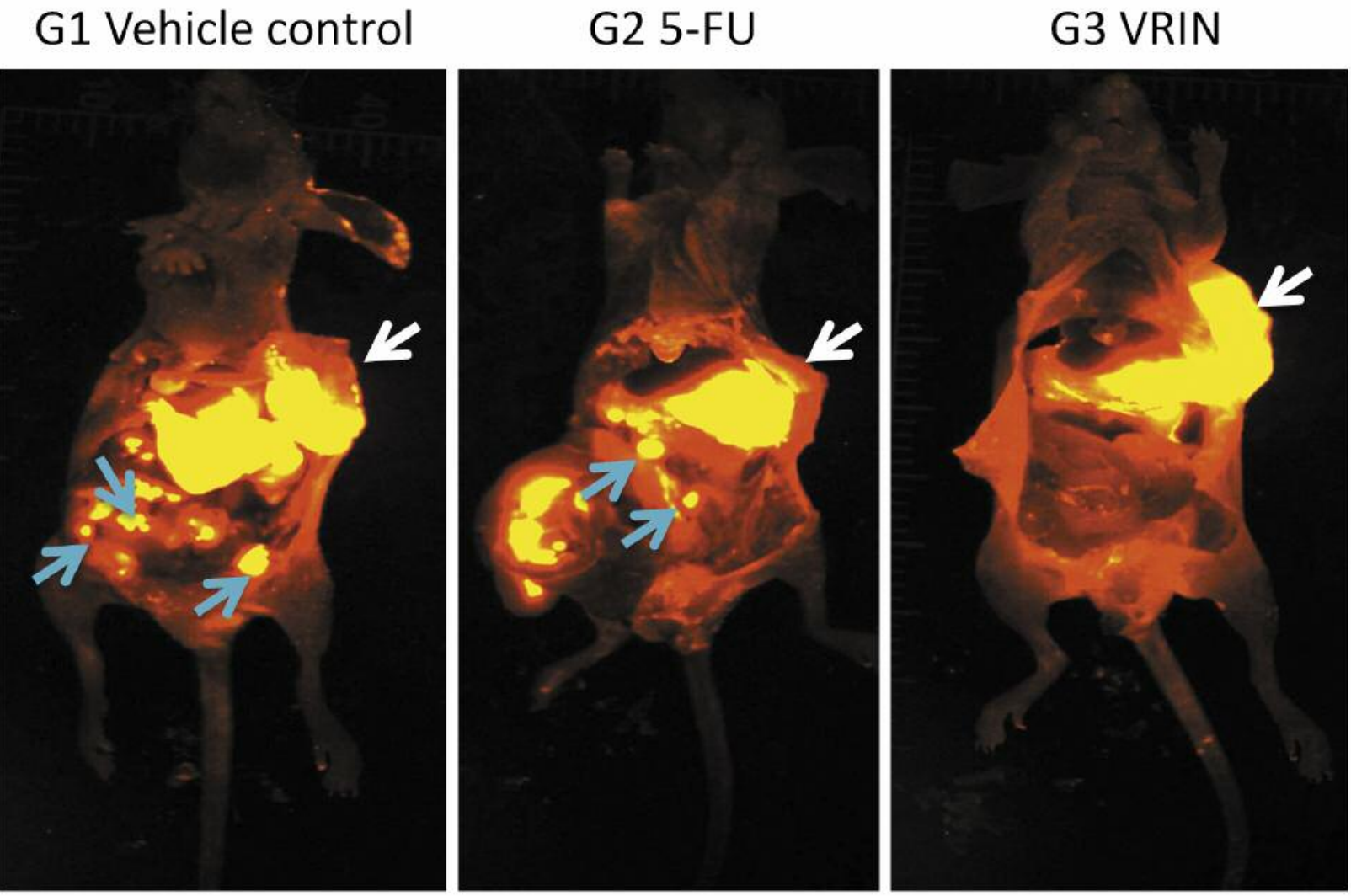

Figure 2. Efficacy of VRIN treatment on metastasis in the NUGC-4-RFP gastric-cancer orthotopic mouse model. Metastasis was identified by open fluorescence imaging at autopsy. Panel depicts representative images in mice treated with vehicle, 5-FU or VRIN. White arrows indicate gastriccancer primary tumor. Blue arrow indicates lymph node metastasis.

\section{Results}

VRIN inhibits tumor growth. Treatments were initiated 9 days after surgical orthotopic implantation of the GC nude-mouse model. Efficacy of VRIN treatment on tumor growth was evaluated by whole-body fluorescence imaging in NUGC-4RFP gastric-cancer orthotopic mouse models. Tumor size was imaged on day 9, 12, 17, 24 and 31 following tumor implantation. Representative fluorescence images and mean tumor volumes are shown in Figure $1 \mathrm{~A}$ and B, respectively. The tumor volumes were significantly reduced in the 5-FU and VRIN groups compared to the untreated control group from day 15 following treatment initiation $(p<0.05)$. As shown in Figure $1 \mathrm{C}$, final tumor weights in the VRIN- and 5-FU-treated groups were also significantly reduced compared to the control group $(p<0.05)$.

VRIN reduced lymphatic metastasis. At the end of the study all animals were sacrificed and metastasis was examined by open fluorescence imaging. Representative open fluorescence images and metastasis rates are shown in Figure 2 and Table I,

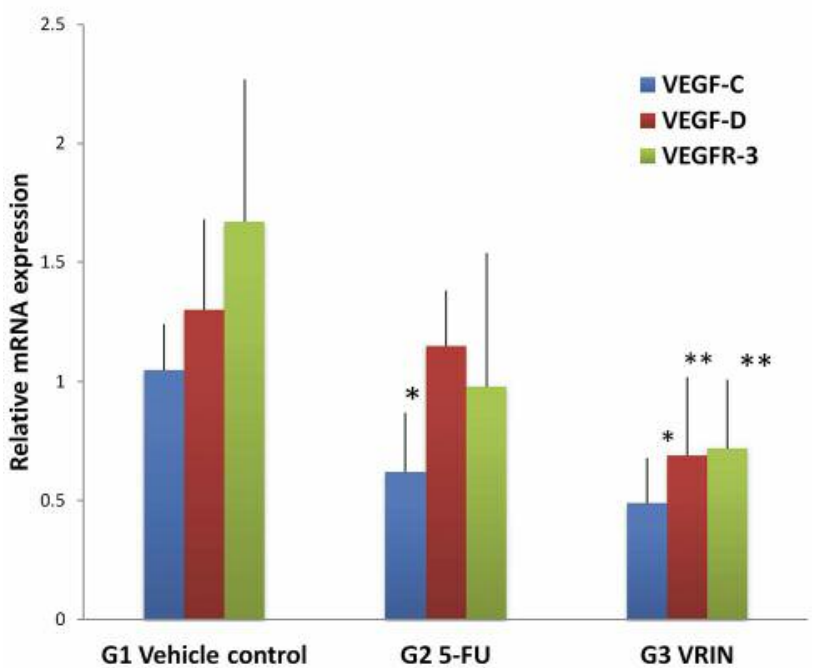

Figure 3. Efficacy of VRIN treatment on mRNA expression of tumor lymphangiogenesis-related factors. mRNA expression of VEGF-C, VEGF-D and VEGFR-3 in mice treated with vehicle, 5-FU or VRIN was detected by real-time quantitative PCR. *p<0.01 and $* * p<0.05$, compared to vehicle control. 


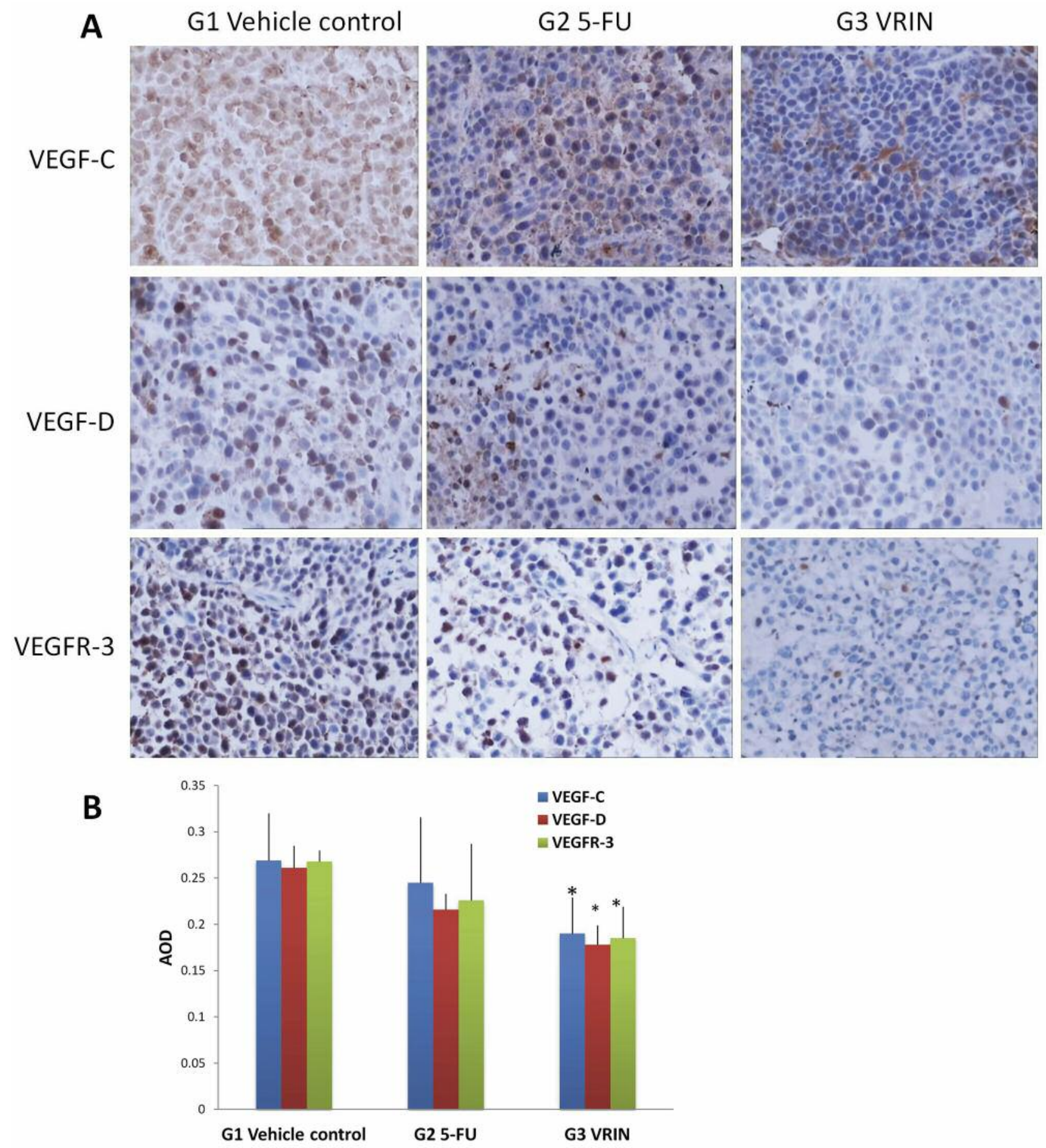

Figure 4. Efficacy of VRIN treatment on protein expression of tumor lymphangiogenesis-related factors. (A) Representative immunohistochemical images for protein expression of VEGF-C, VEGF-D and VEGFR-3 in mice treated with vehicle, 5-FU or VRIN (400x magnification). (B) Quantitation of the expression of VEGF-C, VEGF-D and VEGFR-3 by average optical density $(A O D) .{ }^{*} p<0.05$, when compared to vehicle control.

respectively. The VRIN-treated group showed significantly less lymph-node metastasis $(1 / 8,12.5 \%)$ than the vehicle control $(7 / 8,87.5 \%)$ or 5 -FU-treated $(4 / 8,50 \%)$ group $(p<0.05)$.
VRIN suppress expression of VEGF-C, VEGF-D and VEGFR3. mRNA expression of VEGF-C, VEGF-D and VEGFR-3 in the tumor was detected using real-time PCR. As shown in 
Table I. Efficacy of VRIN and 5-FU treatment on metastasis in the NUGC-4-RFP gastric-cancer orthotopic nude-mouse model.

\begin{tabular}{lcccc}
\hline Group & $\begin{array}{c}\text { No. of animals } \\
\text { per group }\end{array}$ & LN MI & LN MR & $p$-Value \\
\hline Vehicle control & 8 & 7 & $87.5 \%$ & \\
5-FU & 8 & 4 & $50 \%$ & $>0.05^{*}$ \\
VRIN & 8 & 1 & $12.5 \%$ & $<0.05^{*}$ \\
\hline
\end{tabular}

LN, Lymph node; MI, metastatic incidence; MR, metastatic rate. *Compared to vehicle control.

Figure 3, the expression of VEGF-C mRNA in the tumor treated with 5-FU or VRIN was significantly down-regulated compared with the vehicle control $(p<0.01)$. Significantly lower VEGF-D and VEGFR-3 mRNA expression was found in the VRIN-treated group compared with the control group $(p<0.05)$. In contrast, the 5-FU-treated group showed no significant differences for VEGF-D and VEGFR-3 mRNA expression as compared with control group $(p>0.05)$.

Immunohistochemical staining of tumor tissue was performed to assess the effect of VRIN on protein expression of VEGF-C, VEGF-D and VEGFR-3. As shown in Figure 4, positive protein expression of VEGF-C, VEGF-D and VEGFR-3 was present in control tumors. Tumors in the VRIN-treated group showed significantly decreased VEGF-C, VEGF-D and VEGFR-3 protein expression compared with the control group $(p<0.05)$. In contrast, no significant reduction of VEGF-C, VEGF-D and VEGFR-3 protein expression was found in the 5-FU-treated group as compared with the untreated control $(p>0.05)$.

Body weight and toxicity. Clinical observation and body weight measurement of animals during the study were performed to assess the toxicity of VRIN treatment. No physical or behavioral signs that indicated adverse effects due to the treatments were observed in the VRIN- or 5-FU-treated groups. As shown in Figure 5, stable body weight in VRINand 5-FU-treated groups indicated no obvious toxicity.

\section{Discussion}

Ginsenoside $\operatorname{Rg}_{3}$ has been reported to be a relatively safe drug and previously exhibited significant anticancer activity in vitro and in vivo in several types of tumors $(25,26)$. However, its poor aqueous solubility and low oral bioavailability limit its potential clinical use. Yang et al. (27) prepared nano-size ginsenoside $\mathrm{Rg}_{3}$ particle-loaded magnetic human serum albumin nanosheres $\left(\mathrm{Rg}_{3} / \mathrm{HSAMNP}\right)$ to improve its aqueous solubility and bioavailability. $\mathrm{Rg}_{3}$ /HSAMNP combined with magnetic hyperthermia therapy inhibited cell growth and induced apoptosis in HeLa cells in vitro. In the present study we used a new form of

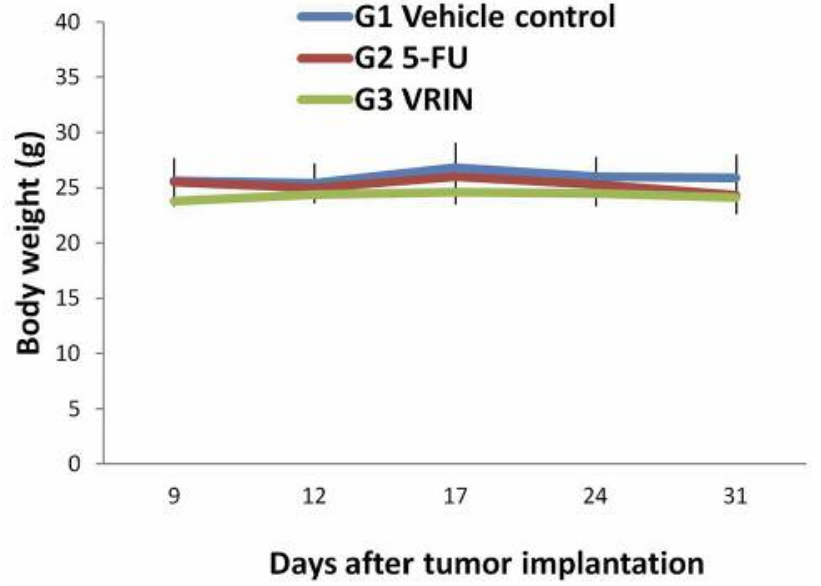

Figure 5. Effect of VRIN treatment on mouse body weight in the NUGC4-RFP gastric-cancer orthotopic nude-mouse model. No significant body weight loss was observed in 5-FU-and VRIN-treated mice.

ginsenoside $\operatorname{Rg}_{3}$ nanoemulsion, to investigate its efficacy in an orthotopic nude-mouse model of human gastric cancer. Our results indicate that this ginsenoside $\mathrm{Rg}_{3}$ nanoemulsion significantly inhibited tumor growth.

In the present study, we used an anti-VEGFR-3 antibodyconjugated ginsenoside $\mathrm{Rg}_{3}$ nanoemulsion to target lymphangiogenesis in the orthotopic gastric-cancer model which has a high frequency of lymph-node metastasis. Consequently, this treatment showed more significant inhibition of lymph-node metastasis than standard chemotherapy with 5FU. Anti-VEGFR-3 antibody can block activation of the corresponding cellular receptor by sequestering the ligand, VEGF-C or VEGF-D. Our study showed that expression of VEGF-C, VEGF-D and VEGFR-3 in the tumor was suppressed by VRIN treatment, while expression of VEGF-D and VEGFR3 in the 5-FU-treated group was not significantly affected. This result indicates the effectiveness of anti-VEGFR-3 antibody on targeting lymphangiogenesis in the orthotopic mouse model of human gastric cancer.

In conclusion, this study shows that the treatment with ginsenoside $\mathrm{Rg}_{3}$-conjugated VEGFR-3 antibody nanoemulsion inhibited tumor growth and reduced lymphatic metastasis (Table I) possibly by suppressing expression of VEGF-C, VEGF-D and VEGFR-3 in an orthotopic mouse model of human gastric cancer. Our study demonstrates the potential of traditional Chinese medicine as an effective targeted treatment for human cancer and the power of orthotopic mouse models for identifying such efficacy (28-34).

\section{Conflicts of Interest}

None of the Authors have a conflict of interest in regard to this study. 


\section{Acknowledgements}

This research was supported by the National Natural Science Foundation of China (No. 81001589, No. 81573656), the Opening Research Project of Jiangsu Key Laboratory of Integrated Traditional Chinese and Western Medicine for Prevention and Treatment of Senile Diseases (No. 2015LNB004), and the Natural Science Foundation of Yangzhou (No. YZ2014063).

\section{References}

1 Siegel RL, Miller KD and Jemal A: Cancer statistics, 2015. CA Cancer J Clin 65: 5-29, 2015.

2 Chen W: Cancer statistics: updated cancer burden in China. Chinese J Cancer Res 27: 1, 2015.

3 Norero E, Bustos M, Herrera ME, Cerda J, González P, Ceroni M, Martínez C, Briceño E, Rojas H, Cártes R, Lopez V, Hidalgo V, Báez S, Caracci M, Viñuela E and Díaz A: Postoperative adjuvant treatment for gastric cancer improves long-term survival after curative resection and D2 lymphadenectomy. Results from a Latin American Center. Euro J Surg Oncol 42: 94-102, 2016.

4 Strong VE, Wu AW, Selby LV, Gonen M, Hsu M, Song KY, Park $\mathrm{CH}$, Coit DG, Ji JF and Brennan MF: Differences in gastric cancer survival between the U.S. and China. J Surg Oncol 112: 31-7, 2015.

5 Takagane A, Terashima M, Abe K, Araya M, Irinoda $\mathrm{T}$, Yonezawa H, Nakaya T, Inaba T, Oyama K, Fujiwara H and Saito K: Evaluation of the ratio of lymph node metastasis as a prognostic factor in patients with gastric cancer. Gastric Cancer 2: 122-128, 1999.

6 Kunisaki C, Shimada H, Takahashi M, Ookubo K, Moriwaki Y, Akiyama $\mathrm{H}$ and Nomura M: Prognostic factors in early gastric cancer. Hepatogastroenterology 48: 294-298, 2001.

7 Nitti D, Marchet A, Olivieri M, Ambrosi A, Mencarelli R, Belluco $\mathrm{C}$ and Lise M: Ratio between metastatic and examined lymph nodes is an independent prognostic factor after D2 resection for gastric cancer: Analysis of a large European monoinstitutional experience. Ann Surg Oncol 10: 1077-1085, 2003.

8 Joukov V, Pajusola K, Kaipainen A, Chilov D, Lahtinen I, Kukk E, Saksela O, Kalkkinen N and Alitalo K: A novel vascular endothelial growth factor, VEGF-C, is a ligand for the Flt4 (VEGFR-3) and KDR (VEGFR-2) receptor tyrosine kinases. EMBO J 15: 290-298, 1996.

9 Jeltsch M, Kaipainen A, Joukov V, Meng X, Lakso M, Rauvala H, Swartz M, Fukumura D, Jain RK and Alitalo K: Hyperplasia of lymphatic vessels in VEGF-C transgenic mice. Science 276: 1423-1425, 1997.

10 Achen MG, Jeltsch M, Kukk E, Mäkinen T, Vitali A, Wilks AF, Alitalo K and Stacker SA: Vascular endothelial growth factor D (VEGF-D) is a ligand for the tyrosine kinases VEGF receptor 2 (Flk1) and VEGF receptor 3 (Flt4). Proc Natl Acad Sci USA 95: 548-553, 1998.

11 Yonemura Y, Endo Y, Fujita H, Fushida S, Ninomiya I, Bandou E, Taniguchi K, Miwa K, Ohoyama S, Sugiyama K and Sasaki $\mathrm{T}$ : Role of vascular endothelial growth factor $\mathrm{C}$ expression in the development of lymph node metastasis in gastric cancer. Clin Cancer Res 5: 1823-1829, 1999.

12 Ishikawa M, Kitayama J, Kazama S and Nagawa H: Expression of vascular endothelial growth factor C and D (VEGF-C and -D) is an important risk for lymphatic metastasis in undifferentiated early gastric carcinoma. Jpn J Clin Oncol 33: 21-27, 2003.

13 Choi YJ, Lee HJ, Kang DW, Han IH, Choi BK and Cho WH: Ginsenoside $\mathrm{Rg}_{3}$ induces apoptosis in the U87MG human glioblastoma cell line through the MEK signaling pathway and reactive oxygen species. Oncology Reports 30: 1362-1370, 2013.

14 Pan XY, Guo H, Han J, Hao F, An Y, Xu Y, Xiaokaiti Y, Pan Y and $\mathrm{Li} \mathrm{XJ}$ : Ginsenoside $\mathrm{Rg}_{3}$ attenuates cell migration via inhibition of aquaporin 1 expression in $\mathrm{PC}-3 \mathrm{M}$ prostate cancer cells. Euro J Pharmacol 683: 27-34, 2012.

15 Chen XP, Qian LL, Jiang $\mathrm{H}$ and Chen JH: Ginsenoside $\mathrm{Rg}_{3}$ inhibits CXCR4 expression and related migrations in a breast cancer cell line. Intl J Clin Oncol 16: 519-523, 2011.

16 Yuan HD, Quan HY, Zhang Y, Kim SH and Chung SH: 20(S)Ginsenoside $\mathrm{Rg}_{3}$-induced apoptosis in HT-29 colon cancer cells is associated with AMPK signaling pathway. Molecular Medicine Reports 3: 825-831, 2010.

17 Zhang C, Liu L, Yu Y, Chen B, Tang C and Li X: Antitumor effects of ginsenoside $\mathrm{Rg}_{3}$ on human hepatocellular carcinoma cells. Molecular Medicine Reports 5: 1295-1298, 2012.

18 Delmas T, Piraux H, Couffin AC, Texier I, Vinet F, Poulin P, Cates ME, Bibette J: How to prepare and stabilize very small nanoemulsions. Langmuir 27: 1683-1692, 2011.

19 Koo OM, Rubinstein I and Onyuksel H: Role of nanotechnology in targeted drug delivery and imaging: a concise review. Nanomedicine 1: 193-212, 2005.

20 Ravi Theaj Prakash U and Thiagarajan P: Nanoemulsion for drug delivery through different routes. Res Biotechnol 2: 1-13, 2011.

21 Koga K, Takarada N and Takada K: Nano-sized water-in-oil-inwater emulsion enhances intestinal absorption of calcein, a high solubility and low permeability compound. Eur J Pharm Biopharm 74: 223-232, 2010.

22 Tadros T: Application of rheology for assessment and prediction of the long-term physical stability of emulsions. Adv Colloid Interface Sci 108-109: 227-258, 2004.

23 Hoffman RM: Orthotopic metastatic mouse models for anticancer drug discovery and evaluation: a bridge to the clinic. Investigational New Drugs 17: 343-359, 1999.

24 Hoffman RM: The multiple uses of fluorescent proteins to visualize cancer in vivo. Nat Rev Cancer 5: 796-806.

25 Zhang Y, Liu QZ, Xing SP and Zhang JL: Inhibiting effect of Endostar combined with ginsenoside $\mathrm{Rg}_{3}$ on breast cancer tumor growth in tumor-bearing mice. Asian Pac J Trop Med 9: 180$183,2016$.

26 Junmin S, Hongxiang L, Zhen L, Chao Y and Chaojie W: Ginsenoside $\mathrm{Rg}_{3}$ inhibits colon cancer cell migration by suppressing nuclear factor kappa B activity. J Tradit Chin Med 35: 440-444, 2015.

27 Yang R, Chen D, Li M, Miao F, Liu P and Tang Q: 20(s)ginsenoside $\mathrm{Rg}_{3}$-loaded magnetic human serum albumin nanospheres applied to HeLa cervical cancer cells in vitro. Biomed Mater Eng 24: 1991-1998, 2014.

28 Wang M, Zhang X, Xiong X, Yang Z, Sun Y, Yang Z, Hoffman RM and Liu Y: Efficacy of the Chinese Traditional Medicinal Herb Celastrus orbiculatus Thunb on human hepatocellular carcinoma in an orthotopic fluorescent nude mouse model. Anticancer Res 32: 1213-1220, 2012.

$29 \mathrm{Hu}$ M, Zhao M, An C, Yang M, Zhang Y, Suetsugu A, Tome Y, Yano $\mathrm{S}, \mathrm{Fu} \mathrm{Y}$, Hoffman RM and Hu K: Real-time imaging of apoptosis induction of human breast cancer cells by the traditional Chinese medicine herb tubeimu. Anticancer Res 32: 2509-2514, 2012. 
30 Zhang Y, Zhang N, Su S, Hoffman RM and Zhao M: Salmonella typhimurium A1-R tumor targeting in immunocompetent mice is enhanced by a Traditional Chinese Medicine herbal mixture. Anticancer Res 33: 1837-1843, 2013.

31 Zhang L, Wu C, Zhang Y, Liu F, Zhao M, Bouvet M and Hoffman RM: Efficacy comparison of traditional Chinese medicine LQ versus gemcitabine in a mouse model of pancreatic cancer. J Cell Biochem 114: 2131-2137, 2013.

32 Zhang L, Wu C, Zhang Y, Liu F, Wang X, Zhao M and Hoffman RM: Comparison of efficacy and toxicity of Traditional Chinese Medicine (TCM) herbal mixture LQ and conventional chemotherapy on lung cancer metastasis and survival in mouse models. PLoS One 9: e109814, 2014.

33 Zhang L, Wu C, Bouvet M, Yano S and Hoffman RM: Traditional Chinese medicine herbal mixture LQ arrests FUCCIexpressing HeLa cells in $\mathrm{G}_{0} / \mathrm{G}_{1}$ phase in 2D plastic, 2.5D Matrigel ${ }^{\circledR}$, and 3D Gelfoam ${ }^{\circledR}$ culture visualized with FUCCI imaging. Oncotarget 6: 5292-5298, 2015.
34 Yin G, Tang D, Dai J, Liu M, Wu M, Sun Y, Yang Z, Hoffman RM, Li L, Zhang S and Guo X: Combination efficacy of Astragalus membranaceus and Curcuma wenyujin at different stages of tumor progression in an orthotopic nude mouse model of metastatic human ovarian cancer. Anticancer Res 35: 31933208, 2015.
Received January 10, 2017

Revised February 16, 2017

Accepted February 20, 2017 